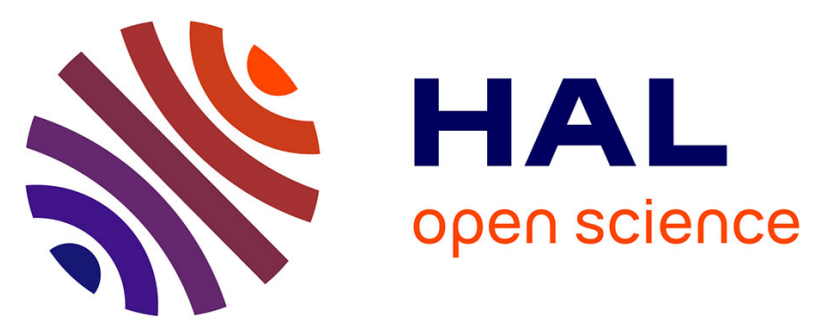

\title{
LA CONCEPTUALISATION EN SOCIOLOGIE: INFLUENCES PARADIGMATIQUES ET IMPLICATIONS METHODOLOGIQUES. L'EXEMPLE DE LA NOTION DE RISQUE DANS LE SPORT
}

\author{
Bastien Soulé, Jean Corneloup
}

\section{To cite this version:}

Bastien Soulé, Jean Corneloup. LA CONCEPTUALISATION EN SOCIOLOGIE: INFLUENCES PARADIGMATIQUES ET IMPLICATIONS METHODOLOGIQUES. L'EXEMPLE DE LA NOTION DE RISQUE DANS LE SPORT. Bulletin de Méthodologie Sociologique / Bulletin of Sociological Methodology, 2007, 93, pp.28-54. halshs-00377066

\section{HAL Id: halshs-00377066 \\ https://shs.hal.science/halshs-00377066}

Submitted on 22 Apr 2009

HAL is a multi-disciplinary open access archive for the deposit and dissemination of scientific research documents, whether they are published or not. The documents may come from teaching and research institutions in France or abroad, or from public or private research centers.
L'archive ouverte pluridisciplinaire HAL, est destinée au dépôt et à la diffusion de documents scientifiques de niveau recherche, publiés ou non, émanant des établissements d'enseignement et de recherche français ou étrangers, des laboratoires publics ou privés. 


\title{
LA CONCEPTUALISATION EN SOCIOLOGIE : INFLUENCES PARADIGMATIQUES ET IMPLICATIONS METHODOLOGIQUES. L'EXEMPLE DE LA NOTION DE RISQUE DANS LE SPORT
}

\author{
par \\ B. Soulé \\ (Centre de Recherche sur les Activités Physiques et Sportives, Université de Caen \\ Basse-Normandie) \\ J. Corneloup \\ (Laboratoire TERRITOIRES, Université Joseph Fourier, Grenoble 1)
}

\begin{abstract}
"Conceptualization in Sociology: Paradigmatic Influences and Methodological Implications"

Abstract: Such a seemingly self-apparent notion as "risky sports" is widely used in social science literature. Through the in-depth analysis of this term, the purpose of this article is to examine a crucial step of the sociological research process: definition of concepts. After having underlined simplifications and paradoxes inherent in the common sense use of this term, we present an overview of its scientific uses and definitions. Some terminological difficulties soon appear, along with differing points of view and plural available conceptualizations. The critical, structural, subjectivist, culturalist, and systemic approaches can thus be differentiated. The choice made between these options entail methodological consequences that are also discussed. Two epistemological positions can eventually be adopted. On one hand, one can be in favour of the abandon of such a category, on account of its intrinsic shakiness, and because it is merely impossible to reach by consensus the one and only definition of "risky sports". On the other hand, one can acknowledge the polythetic nature of this term. The latter will then be easily observed through the specific constructions of "risky sports", as a scientific object, according to each research problematics.

Key-words: Conceptualization, Hazards, Risky Sports.
\end{abstract}

Résumé : Cet article propose une réflexion sur l'acte de définition en sociologie, à 
travers l'analyse approfondie de la notion de «sport à risque ». Fréquemment mobilisée dans la littérature en sciences sociales, celle-ci semble généralement aller de soi, notamment parce qu'elle repose sur quelques sous-entendus empruntés au sens commun. Après avoir souligné les simplifications et paradoxes inhérents à cet usage spontané de l'appellation «sport à risque», nous avons entrepris un recensement de ses utilisations et définitions scientifiques. Apparaissent dès lors certaines difficultés terminologiques, des divergences de vue, et la pluralité des conceptualisations disponibles. Les approches critique, structurelle, subjectiviste, culturaliste et systémique ont ainsi pu être différentiées. Les conséquences méthodologiques des choix notionnels opérés par les chercheurs sont également présentées. Au terme de cette analyse, deux positions peuvent être adoptées: renoncer à l'usage d'une catégorie jugée fragile, en constatant l'impossibilité de parvenir à une définition sinon générique, partagée du risque sportif ; ou admettre le caractère polythétique de cette appellation, se traduisant par des constructions spécifiques de l'objet «sport à risque » en fonction de chaque problématique de recherche.

Mots clés : Conceptualisation, Risque, Sports à risque. 


\section{INTRODUCTION}

Depuis une vingtaine d'années, rares sont les analyses des pratiques sportives contemporaines ne soulignant pas une certaine radicalisation de l'engagement corporel de leurs adeptes. La mise sous forme risquée et aventureuse des activités sportives, qui constitue, selon Le Breton (2002) et Stranger (1999), un trait des sociétés occidentales, est ainsi soulignée par de nombreux chercheurs en sciences sociales ${ }^{1}$.

Bien que concurrencée depuis la fin des années 1990 par la notion floue de «sport extrême » (Soulé, 2006), l'appellation « sport à risque » bénéficie d'un franc succès pour quiconque souhaite faire état de ces expositions accrues aux dangers. Cet article propose une réflexion terminologique approfondie autour de cette catégorie d'analyse. En effet, si certaines modalités de pratique exacerbent à l'évidence l'expérience du vertige, le test des capacités de résistance des sportifs, voire la confrontation effective à des dangers, le fréquent recours à la notion de «sport à risque » entraîne son lot de généralisations abusives et d'assimilations simplificatrices. Le simple bon sens suffit dans un premier temps à se convaincre des ambiguïtés terminologiques soulevées par cette notion : certaines activités sportives sont-elles sans danger? Les risques sportifs sont-ils apparus simultanément à la création de cette appellation ? Doit-on, pour caractériser une activité sportive de risquée, se baser sur la fréquence des accidents, la criticité des traumatismes effectifs ou potentiels, les représentations du grand public, les traits caractéristiques de l'activité, son environnement, ou encore le ressenti émotionnel des pratiquants?

Ces difficultés de définition, tout comme le statut ontologiquement problématique de la catégorie de «sport à risque», sont rarement évoqués. Pourtant, selon Majastre (1990), la notion de «sports à risque», trop communément et trop immédiatement comprise, est l'objet de nombreux malentendus. Certaines voix s'élèvent d'ailleurs contre son usage inconsidéré (Collard, 1998 ; Seigneur, 2004). Afin d'y remédier, il semble nécessaire de clarifier le sens des allusions aux « sports à risque » et au risque sportif, de recenser les formalisations entreprises, les aspects sur lesquels elles mettent l'accent, ainsi que leurs inscriptions paradigmatiques $^{2}$. Tout en se gardant de prétendre parvenir à la bonne définition, l'enjeu consiste à conserver une certaine maîtrise théorique de cette notion. Cette maîtrise est inenvisageable sans mémoire conceptuelle, c'est-à-dire sans une

\footnotetext{
${ }^{1}$ Midol (1993), Pociello (1995), Kay et Laberge (2002), Rinehart et Sydnor (2003), Baddeley (2003), etc.

${ }^{2}$ Nous prenons ici le parti d'affirmer que des paradigmes structurent la pensée sociologique contemporaine, bien qu'une telle opinion épistémologique ne fasse pas l'unanimité. Selon Babbie (1998), dans le champ des sciences sociales, contrairement à ce que Kuhn (1972) observe ailleurs, les paradigmes ne se succèdent pas au fil des découvertes : ils gagnent et perdent en popularité, mais sont rarement abandonnés, et encore moins supplantés par une quelconque "révolution scientifique ». Cette absence de révolution théorique « faisant table rase du passé » pousse Passeron (2006) à affirmer que parler de paradigmes en sociologie est déplacé.
} 
connaissance du passé et des origines du terme, lesquels contribuent à définir ses pertinences opératoires ou argumentatives actuelles (Passeron, 2006).

Par ailleurs, l'occasion est belle d'élargir la réflexion à la conceptualisation en sociologie, enjeu majeur de cette discipline confrontée à sa propre contemporanéité. Le choix des mots en sciences sociales est en effet particulièrement délicat, les notions mobilisées ayant presque toujours été préalablement utilisées, selon des acceptions fort diverses, y compris dans le langage courant (Bajoit, 2003). Cette multiplicité des emplois constitue selon Passeron (2006) un des principaux obstacles s'opposant à une délimitation claire des concepts en sociologie.

Enfin, cette approche constitue une invitation à repenser les liens entre théorie et méthodologie. La définition choisie par le chercheur, dépendante de son paradigme de référence, engage en effet des catégories de lecture qui orientent la construction des outils méthodologiques, et plus généralement la manière d'observer les faits.

\section{DIFFICULTES INHERENTES A LA CONCEPTUALISATION EN SCIENCES SOCIALES}

Se saisir sociologiquement d'un objet implique une reconstruction qui ne va pas de soi, du fait de la double herméneutique auxquelles les sciences sociales sont sujettes (Giddens, 1987) : elles doivent non seulement construire leur objet, mais également tenir compte des diverses interprétations (de caractère spontané ou savant) préexistant à l'observation (Duchastel et Laberge, 1999). Circonscrire les concepts avec précision est donc délicat, voire impossible si l'on en croit Passeron (2006), qui souligne une conséquence notable de cette difficulté : le large espace interprétatif créé est propice à la construction de davantage de concepts, d'où une certaine «inflation terminologique ». Pour en revenir aux «sports à risque », les difficultés conceptuelles et les risques d'approximation terminologique sont accentués par la diffusion de ce terme dans le langage courant, susceptible de générer un effet pervers consistant à considérer cette catégorie du sens commun suffisamment « parlante » pour ne pas s'embarrasser de coûteuses précautions.

Au-delà de cette première spécificité, si la langue sociologique est «encombrée », s'apparentant même à une forme d'anomie conceptuelle, c'est aussi parce «qu'il n'existe pas d'énoncé sociologique qui échappe aux limitations de sens et d'audience que l'état conflictuel et éclaté du champ théorique lui prescrit» (Passeron, 2006, 93). Autrement dit, le fait que «les mots s'ajoutent aux mots» témoigne de l'éclatement paradigmatique de la sociologie.

Enfin, travailler sur le risque revient à s'aventurer sur un terrain conceptuel glissant. Renn (1998) n'en parle-t-il pas comme d'une «notion problème »? Jardine et Hrudey (1997) soulignent l'absence de définition consensuelle de ce terme. Pour preuve, lorsque la prestigieuse Society for Risk Analysis a été créée aux Etats-Unis, un comité fut mis en place pour définir cette notion. Après quatre années de travail, 
ce comité «jeta l'éponge », concluant à l'impossibilité de fournir une définition générique et jugeant préférable de laisser chaque chercheur définir le risque en fonction de ses préoccupations (Kaplan, 1997). A propos des «sports à risque », il faut donc s'attendre à une certaine pluralité, inhérente à la diversité des questions de recherche.

\section{LES IMPLICITES DE LA NOTION DE «SPORT A RISQUE »}

La familiarité que chacun entretient avec la notion de «sport à risque » s'accompagne généralement de sous-entendus dont il convient de faire état et de comprendre les origines.

\section{Risque et danger : des notions fréquemment confondues}

Jackson et al. (1972) ont souligné de longue date la multidimensionnalité du risque, en vertu de laquelle l'exposition à l'incertitude peut être physique, mais aussi financière, éthique ou encore sociale. Pourtant, les «sports à risque » sont implicitement et presque exclusivement considérés sous l'angle des dangers corporels dont ils sont porteurs. Sur un plan strictement lexical, évoquer une activité risquée ne signifie pourtant pas grand-chose. La principale limite de la notion de «sport à risque » est ainsi soulignée : la nature de la menace, le type d'événement redouté et l'impact éventuel sont rarement mentionnés. Il est sousentendu qu'il s'agit d'écueils corporels.

Un bon moyen d'éviter ce flou consiste à séparer risque et danger. Confondre ou assimiler ces deux notions est en effet une erreur conceptuelle, car bien qu'étroitement liées, elles revêtent des significations fort différentes. Le danger constitue une menace, un événement dommageable susceptible de se produire (chute, collision, épuisement, déshydratation, etc.). Cet évènement peut à son tour entraîner un impact plus ou moins conséquent (contusion, entorse, perte de conscience, fracture, décès, etc.). Le danger ne constitue donc rien de plus que la possibilité qu'un accident survienne, caractérisé par la nature de l'événement et la gravité du dommage éventuel (Lavigne et al., 1989). Dans le domaine sportif, de nombreux dangers peuvent être distingués, parfois étroitement liés à une activité spécifique : entorses de genou pour le football féminin et le ski alpin ; fractures du poignet et/ou du coude parmi les snowboarders ; mal aigu des montagnes et hypothermie lors de la pratique de l'alpinisme ; effets à long terme des produits dopants utilisés par certains sportifs; etc.

Les risques, pour leur part, ne constituent que la façon d'appréhender ces dangers (Kates et Kasperson, 1983) : quelles sont les chances pour que l'événement se matérialise? Pour quelle(s) raison(s) ? Et avec quelles conséquences ? Ces suppositions et évaluations subjectives sont plus ou moins renseignées, parfois 
fantaisistes, souvent ancrées dans des croyances, et plus rarement issues d'approches scientifiques. Elles produisent une variété de points de vue à propos d'un seul et même danger, le risque n'étant qu'une façon, parmi d'autres, d'appréhender et de se représenter le danger (Joffe, 2003). C'est une construction de l'esprit (Beck, 1999), de même qu'un produit culturel, chaque société établissant ce qui est dangereux, quels sont les risques acceptables ou valorisés ainsi que ceux qu'il est déraisonnable d'affronter (Douglas, 1985).

Retenons que le risque est avant tout une manière de considérer le danger, luimême fort variable selon les activités sportives. La fréquente assimilation des notions de risque et danger contribue à l'impression de flou entourant la catégorie de « sport à risque».

\section{Enumérer plutôt que définir : une solution de facilité fréquemment adoptée}

Une autre source d'imprécision réside dans le fréquent recours à l'énumération, au détriment d'un réel travail de définition. Moult activités sportives sont ainsi étiquetées «à risque»: saut à l'élastique, chute libre, B.A.S.E. jump, parapente, escalade, alpinisme, V.T.T., roller, skateboard, rafting, snowboard, freeride, sports de combat, raids-aventure, surf, voile en solitaire, etc. En toute rigueur, de telles énumérations (Schrader et Wann, 1999 ; Lupton, 1999) ne constituent pas des tentatives de clarification terminologique. Se référer à ce type d'inventaire est même une manière de contourner la plus élémentaire des tâches conceptuelles. Ce faisant, de nombreux chercheurs se satisfont de l'appropriation de descriptions distordues de la réalité, savamment orchestrées par les médias (Kusz, 2003) et prestataires de services sportifs (Seigneur, 2004). On aboutit à un amalgame artificiel et peu cohérent de vertige, de sensations, d'aventure, de violence, de vitesse, d'acrobaties, de jeu avec la gravité, d'endurance, de courage et d'environnements hostiles.

De telles utilisations de la notion de « sport à risque » fournissent un certain confort intellectuel, en permettant de ne pas se plier à une délicate et fastidieuse entreprise conceptuelle. Cependant, ces «définitions par énumération» contribuent à entretenir et renforcer une ambiguïté lexicale fondamentale, semblable à celle observée à propos des notions de «sport extrême » (Soulé, 2006) et d'aventure sportive (Seigneur, 2004).

\section{La priorité accordée à la gravité des accidents sportifs}

Classiquement, le risque est défini comme le produit de la probabilité d'occurrence d'un événement et de ses conséquences néfastes. Pourtant, la désignation des activités sportives risquées passe plus ou moins sous silence le premier élément, au profit du second. Cela n'a rien d'étonnant: les spécialistes de la perception des 
risques ont observé de longue date que lorsque les individus évaluent l'importance des menaces inhérentes à certaines activités, ils sont davantage sensibles aux conséquences des risques encourus qu'à l'estimation de leur fréquence d'occurrence (Kunreuther et Slovic, 1996; Peretti-Watel, 2001). Les enquêtes concernant le domaine sportif le confirment : la désignation des sports les plus risqués se fait par rapport à la gravité des écueils corporels envisagés, davantage qu'en référence à leur probabilité d'occurrence (Collard, 1998 ; Mun, 2004).

Plusieurs sports ainsi qualifiés de risqués ne sont pas des sports impliquant beaucoup d'accidents (Majastre, 1990). Inversement, les pratiques sportives traditionnelles et/ou compétitives, reléguées au second plan dans ce type d'enquête de perception des risques, constituent le gros de l'accidentologie sportive (Arènes et al., 1998).

\section{Les risques sportifs : le produit de prises de risque davantage que des risques subis ?}

Un dernier sous-entendu associe tacitement «sports à risque » et prises de risque. La prise de risque consiste en une décision opportuniste de s'exposer au danger (Hahn et al., 1994) : afin d'atteindre un bénéfice d'ordre quelconque, l'acteur prend plus de risques que la situation ne l'impose. A l'inverse, le péril, ou risque subi, est censé être soustrait au contrôle de l'individu. On prend un risque alors que l'on subit un péril. Le sens commun a fortement tendance à assimiler les «sports à risque » et les prises de risque volontaires. Pourtant, on peut à l'évidence s'exposer involontairement au danger en pratiquant la randonnée ou un sport collectif. La distinction entre prises de risque et risque subi, nous paraissant aller de soi, est en fait socialement construite (Douglas, 1987). De plus, il existe des stratégies commerciales d'effacement ou d'édulcoration des risques qui tendent à minimiser les dangers liés à certains environnements (y compris quand ils sont dangereux « par essence », comme le milieu montagnard) (Bozonnet, 1992) et à faire la promotion d'activités sportives prétendument rendues sûres du fait d'une irréprochable gestion des risques (y compris dans le cadre d'activités accidentogènes comme le canyonning ou l'alpinisme) (Palmer, 2002). Parler automatiquement de prise de risque est dès lors abusif, tant les pratiquants peuvent être amenés à concevoir la fréquentation de tels environnements et l'engagement dans ces activités comme étant dépourvus de danger.

\section{Synthèse : une vision particulière des « sports à risque »}

Ces remarques propédeutiques permettent de souligner l'implicite qui se glisse derrière la notion de «sport à risque », et de mieux comprendre pourquoi, à l'évocation de cette appellation, vient presque spontanément à l'esprit un type bien 
particulier d'engagement corporel : la pratique d'activités de « loisir sportif $»^{3}$, hors cadre institutionnel et en plein air, dans un environnement propice aux accidents, théâtre de prises de risque pouvant entraîner des blessures graves ou la mort. Dans le cadre de cette analyse notionnelle, une précaution s'impose par conséquent : il importe de dépasser les aspects purement lexicaux pour aborder le terrain de la construction sociale et médiatique de la catégorie de « sport à risque ».

Cette appellation constitue en fait un terme ad hoc, qui consiste à spécialiser l'usage d'une expression dont l'emploi est fréquent dans l'intercompréhension quotidienne ${ }^{4}$. Cette simplicité d'emprunt autorise souvent le contournement d'une énonciation des principes de définition (Passeron, 2006); dès lors, les limites conceptuelles de ces objets pré-construits sont fréquemment outrepassées, quand bien même l'inconsistance guette.

\section{LES CONCEPTUALISATIONS DU RISQUE SPORTIF ET/OU DES SPORTS A RISQUE}

Cinq perspectives conceptuelles (critique, structurelle, culturaliste, systémique et subjectiviste) revendiquant plus ou moins fortement leur inscription dans un courant dominant de la sociologie sportive ont été identifiées. On retrouve ainsi les oppositions classiques entre sociologies dénonciatrice, individualiste, structuraliste, systémique et post-moderne ou sensible. Sans surprise, les approches notionnelles recensées dépendent donc de la position de leurs auteurs au sein du champ sociologique.

Par ailleurs, les écrits convoqués revêtent un caractère hétérogène, en raison des différentes intentions de leurs auteurs. En effet, certains s'assignent clairement pour objectif de faire passer les «sports à risque» du statut de notion (connaissance vague, abstraite, immédiate et plus ou moins confuse) à celui de concept ; d'autres n'abordent cette question terminologique que préalablement à leur questionnement principal (l'engagement dans des pratiques sportives qualifiées de risquées, la perception des risques sportifs, la gestion des risques sportifs, etc.). Parmi ces derniers, il faut encore distinguer ceux qui entendent véritablement construire leur objet et ceux qui ne semblent se plier à cet exercice notionnel que par obligation académique, "parce qu'il faut définir». Le Breton (2002) place ainsi sous le vocable d'activités sportives à risque celles qui impliquent une incertitude relative, la confrontation à un danger réel ou imaginaire mettant en jeu l'intégrité physique. Plongée, alpinisme et parapente sont cités en exemple, mais bien d'autres activités sportives (pour ne pas dire toutes) satisfont à ces critères peu discriminants.

\footnotetext{
3 « Les disciplines qu'on appelle sports à risque sont des pratiques de loisir » (Penin, 2004a).

4 Des exemples d'autres notions ad hoc : jeunesse, innovation, mass-media, audio-visuel... Caractérisées par une forme de consensus définitionnel, dans et en dehors du champ sociologique, elles trahissent selon Passeron (2006) l'appartenance à une sociologie plus spontanée que scientifique.
} 
Pour résumer, en vertu de la double pluralité évoquée (en termes d'approches et d'objectifs), les chercheurs mis à contribution n'abordent les «sports à risque »ni sous le même angle, ni en référence aux mêmes théories et paradigmes. Du coup, au sein du paysage terminologique dressé, les similitudes et complémentarités sont aussi nombreuses que les contradictions et incompatibilités. Pour permettre une compréhension affinée de la dynamique du champ notionnel qui se dessine, il s'avèrera donc nécessaire d'expliciter le contexte intellectuel des efforts de définition décrits, à travers un bref exposé des préoccupations scientifiques de leurs auteurs.

Enfin, ces partis-pris conceptuels et théoriques ne sont pas sans incidence sur les analyses empiriques. La manière dont sont envisagées la relation avec le terrain et la méthode d'enquête dépend en effet du positionnement théorique et de l'approche notionnelle du risque, ces trois éléments faisant système. Dit autrement, certaines conceptualisations «désignent» une démarche méthodologique (Passeron, 2006). Cette relation sera abordée pour chaque type de définition présenté.

\section{Les « sports à risque » : un pléonasme ?}

Les tenants d'une sociologie critique du sport prennent le contre-pied de la vision dominante des risques sportifs, exposée dans l'introduction et la première partie de cet article. Selon eux, les risques corporels sont intimement liés à la modernité sportive, et non à des évolutions conjoncturelles des modalités de pratique (Vassort, 2003).

\section{Le risque : un invariant protéiforme de la pratique sportive}

Dans une perspective radicale, Brohm (1986) considère toute pratique sportive ${ }^{5}$ comme physiquement risquée. En s'appuyant sur des indicateurs quantitatifs soulignant la fréquence des lésions et blessures dues à la pratique sportive traditionnelle, ce dernier affirme sa thèse de l'aliénation du sportif par sa pratique et dénonce les dérives de la pratique compétitive. «L'accident sportif (blessures et mutilations graves, lésions irréversibles, traumatismes physiologiques profonds, morts brutales, casses en tous genres, drames de la compétition) n'est évidemment pas le résultat d'une mystérieuse fatalité ou de la glorieuse incertitude du sport (...) mais bel et bien la conséquence inéluctable, à terme, du jeu pervers avec l'urgence mortelle, la compulsion mortifère, la pression de la réussite coûte que coûte » (Brohm, 1987, 50). Baudry résume les tenants du paradigme de l'aliénation en affirmant que « l'excès, dans le domaine sportif, n'est pas exceptionnel mais révèle un système tout entier: l'ordre même d'une rentabilité qui suppose, impose, la défonce de l'individu » (Baudry, 1991, 87). Il s'éloigne toutefois occasionnellement de cette ligne de conduite lorsqu'il cède à la tentation de l'énumération, listant un ensemble de pratiques sportives à risque («sports de glisse, de précipitation, de

\footnotetext{
${ }^{5}$ Le sport dont il est question est institutionnalisé, codifié, réglementé, tourné vers la comparaison des performances, la désignation du champion et la poursuite du record.
} 
chute, de vol, sports de maîtrise des éléments, sports de vitesse, etc. ») (Baudry, 1991, 58), révélatrices à ses yeux d'un rapport excessif et extrême au corps.

\section{Commentaire critique et implications méthodologiques}

A travers le prisme critique, le sport est essentiellement envisagé sous l'angle de ses méfaits. En couplant cette posture intellectuelle tranchée à une définition très restrictive du sport, les sociologues cités supra coupent court à toute velléité conceptuelle. Sport et risque sont tout simplement indissociables, car structurellement liés. Baudry mis à part, les auteurs cités ci-dessus se refusent à envisager l'existence, et incidemment la définition de «sports à risque ». Pour une raison simple : à leurs yeux, tous les sports sont risqués.

Sur le plan empirique, ces travaux se bornent à une analyse relativement superficielle, et rarement contradictoire, de quelques statistiques d'accidentologie soigneusement choisies. Les supputations autour de ces données de seconde main sont du reste fréquentes. Baudry observe pour sa part la manière dont les médias et publicitaires façonneraient les engagements corporels contemporains. Dans les deux cas, l'objet est de valider une thèse préexistante : l'aliénation des sportifs. Ce postulat de départ explique l'absence de réel travail de terrain auprès des sportifs eux-mêmes : aliénés et dominés, ces derniers, par définition, ne sont guère en mesure de fournir d'explication pertinente au sociologue, seul à même de dénoncer des mécanismes sociaux échappant au tout-venant.

\section{Le danger inhérent à la structure des activités sportives}

Plusieurs auteurs ont tenté de répondre à la question suivante : quelles sont les activités sportives présentant des risques particulièrement marqués de blessure ? L'objectif sous-jacent à un tel questionnement consiste à désigner, en adoptant un raisonnement rigoureux, des sports plus dangereux que les autres, afin de ne pas parler dans le vide et sans raison de « sports à risque ». L'existence de ces derniers constitue donc un axiome de départ.

Les « sports à risque » : des activités engendrant des écueils corporels conséquents

Bouet présente dès 1968 une classification des jeux sportifs selon les risques qu'ils recèlent. Bien que tenant compte de la fréquence des accidents, celle-ci est essentiellement basée sur la magnitude estimée de leurs potentiel traumatique. Une triple répartition est proposée : «les sports en lesquels le risque corporel est fondamental et où il fait, en une certaine mesure, partie intrinsèque de l'enjeu impliqué par leur pratique (activités de pleine nature et sports mécaniques) (...) Les sports en lesquels le risque corporel est plus ou moins présent, sous forme, généralement, de la possibilité d'accidents relativement peu graves, et ne constituant pas une dimension essentielle de l'activité sportive en question (gymnastique aux agrès par exemple) (...) Les sports en lesquels le risque corporel 
peut être considéré comme très réduit ou presque nul (le tennis par exemple) » (Bouet, 1968, 127-128).

Cette démarche est poussée à son paroxysme par Penin (2004b), lequel qualifie les «sports à risque »d'activités où la mort n'est pas seulement symbolique ou euphémisée, mais envisageable « en cas d'erreur technique ou matérielle ». C'est la mise en jeu de la vie, et pas seulement de l'intégrité corporelle du pratiquant (présente dans toute activité), qui constitue à ses yeux la véritable variable discriminante. Les indicateurs des «sports à risque» sont dès lors ceux de l'accidentologie, qui désigne l'alpinisme et le parapente comme les activités sportives à risque par excellence ${ }^{6}$ (Penin, 2004a). Rotillon (2003) parle en des termes comparables des pratiques sportives «extrêmes ». Selon lui, l'alpinisme constitue un "sport extrême », du fait du rapport définitif entretenu avec le danger (la vie est mise en jeu), contrairement à l'escalade où « tout est fait pour éliminer le risque mortel » (Rotillon, 2003, 149).

\section{L'accent mis sur les facteurs de risque}

Parlebas (1981) considère lui aussi que c'est la structure $^{7}$ des sports qui prédétermine le niveau de risque pour ses adeptes. Son propos se centre cependant sur les facteurs de risque, là où Bouet et Penin mettent l'accent sur l'étendue des dégâts envisageables. Lorsqu'elle est liée à l'intégrité physique des pratiquants, l'incertitude peut être constitutive de danger. Trois sources d'incertitude sont mentionnées. La première concerne les interactions motrices dans les sports où le contact physique est indissociable de la logique interne. Chacun tentant de rendre ses intentions les plus opaques possibles, elle peut déboucher sur des contacts physiques plus ou moins rudes. L'opposition peut être duelle (sports de combat) ou collective (sports collectifs). La deuxième source d'incertitude est liée aux limites personnelles de l'individu : il y a alors mise à l'épreuve du corps, qui devient adversaire de la volonté (pratiques d'endurance et ascétiques). Les caractéristiques du milieu constituent la troisième source d'incertitude. C'est le «pôle de la sauvagerie ». L'incertitude met alors à l'épreuve la perception du pratiquant et ses facultés motrices. L'incomplétude de l'information produit une part d'aléa et il y a menace à plus ou moins long terme de l'intégrité corporelle (voire de la vie) du pratiquant.

Defrance (1983) s'intéresse lui aussi moins à l'amplitude des écueils corporels qu'à leur nature. Il affirme de manière presque similaire à Parlebas que l'organisme est exposé à deux types de danger plus ou moins présents selon les disciplines : «le risque d'épuisement ou de rupture dans l'effort lui-même et la menace d'un choc

\footnotetext{
${ }^{6}$ Il est tentant d'essayer de définir les « sports à risque » en s'en tenant aux faits, c'est-à-dire à leur accidentologie. Cependant, si quelques pratiques sont relativement bien renseignées sous cet angle (ski alpin, alpinisme), les données statistiques manquent pour entreprendre une rigoureuse comparaison inter-sports (Bourdeau, 1996 ; Kervern et Rubise, 1991).

7 Entendue comme la manière dont les traits de logique interne de ce sport (rapport à l'environnement, interaction entre les joueurs, système de réussite, instrument technique utilisé, temporalité, etc.) sont agencés entre eux (Collard, 1998).
} 
avec l'élément ou l'adversaire par rapport auquel l'activité sportive se joue » (Defrance, 1983, 317). Il distingue cependant deux types de violence : dans les sports de combat, la violence s'exerce avec certitude par le biais d'une action humaine destructrice (il y a bien incertitude lors de chaque assaut, mais pas sur la somme de violence exercée lors du combat) ; dans les sports de nature, a contrario, la destruction n'est pas intentionnelle mais seulement possible et accidentelle.

\section{Une tentative de synthèse entre incertitude de la situation et enjeux corporels}

Collard (1997) a cherché à affiner la formalisation entreprise par Parlebas. A ses yeux, pour qu'il y ait risque, une double condition est nécessaire. Primo, le joueur doit être amené à se dessaisir du contrôle total de la situation. Dans cette optique, l'incertitude, génératrice de processus stochastiques, devient fondatrice. La natation sportive, les courses en couloir ou la gymnastique ne peuvent en conséquence être considérées comme des «sports à risque ». Au contraire, en boxe ou en football, même entraînés de manière optimale, les pratiquants se trouvent en situation d'information imparfaite. Secundo, il faut que le sportif impliqué ait quelque chose à perdre, en l'occurrence son intégrité corporelle. Dans les pratiques de pleine nature, cette donnée ferait intrinsèquement partie du jeu puisque l'échec d'une stratégie motrice peut se traduire par un accident corporel. La conservation de l'intégrité corporelle misée en début de partie constitue selon Collard la solution du jeu avec la nature. Cette double condition permet selon cet auteur d'éviter une perte de la dénotation distinctive du concept, et de classer objectivement certaines pratiques comme des « sports à risque ».

\section{Commentaire critique et implications méthodologiques}

La mise en évidence de déterminants issus de la structure des pratiques est une perspective légitime et séduisante. Elle présente en outre l'avantage de n'exclure aucune pratique sportive a priori. Toutefois, s'engager trop loin dans cette voie terminologique essentialiste peut donner une teneur normative à l'effort de définition.

Dans cette perspective, les traits caractéristiques d'un sport sont censés déterminer la présence ou l'absence de risques. Par conséquent, la manière dont la logique interne d'une activité est décrite est primordiale. L'option choisie par Collard semble dès lors simpliste, puisqu'il met essentiellement à contribution les règlements sportifs. Il y aurait ainsi un enjeu corporel en boxe mais pas en rugby, puisque dans la seconde activité, malgré la rudesse des contacts, une agression serait sévèrement punie. Howe (2001) a pourtant démontré le caractère accidentogène et traumatisant du rugby. A l'inverse, les activités de nature sont décrites comme des pratiques sportives à risque, dans la mesure où la finalité du jeu serait de ne pas se blesser et de revenir indemne.

D'autres critiques peuvent être formulées: quid du niveau d'expertise du pratiquant, décrit par Parlebas (1981) comme un élément déterminant du degré de risque d'une situation sportive ? Quid des différentes modalités de pratique 
identifiées par de nombreux sociologues au sein d'une même discipline ? Au regard de l'étude de Penin (2004a), plus que dans la discipline sportive elle même, c'est essentiellement dans les «façons de faire » que se prennent (ou non) des risques.

Enfin, en excluant le sens investi par les sujets lors de leur pratique, tout comme les vécus et perceptions individuels, Parlebas et Collard s'en tiennent à caractériser certaines activités de dangereuses. Le passage du danger au risque impliquant la présence d'un filtre interprétatif, il n'est pas question de risque à proprement parler. Ce qui compte pour qui entend mobiliser la notion de risque en sciences sociales, ce ne sont donc pas les caractéristiques structurelles de la situation sportive, telles qu'elles ont été « objectivées » par l'analyste, mais le regard porté sur elles et la manière de les appréhender. Autrement dit, les situations risquées qui sont décrites n'ont pas d'existence propre.

Ces réflexions traduisent une forte volonté de respectabilité scientifique. Comme dans l'approche critique, les pratiquants sont évacués du travail de définition, puisque c'est en fonction de certains traits structurels du danger (probabilité d'occurrence du phénomène, gravité des conséquences, type d'accident) que la catégorisation «à risque » s'opère. Il est intéressant de constater que certains chercheurs inscrits dans cette perspective voient leurs échafaudages conceptuels contredits par leurs propres enquêtes. Ainsi, les questionnaires distribués par Collard à ses étudiants, sur le mode des enquêtes psychométriques réalisées par Slovic outre-atlantique, montrent que les individus sont avant tout sensibles aux conséquences des menaces qu'à leur probabilité d'apparition. Toutefois, plutôt que de revenir sur une définition inaltérable, un biais cognitif est évoqué, explicatif du décalage entre une illusoire évaluation rationnelle des risques et la désignation effective des sports à risque. L'objectivation du chercheur prévaut sur les données de terrain.

\section{Les risques sportifs : un complexe de situations vécues et d'expériences individuelles}

Les auteurs mis à contribution dans cette partie cherchent à comprendre la manière dont l'individu se représente et appréhende in situ la situation dangereuse. Le changement de perspective est radical vis-à-vis des conceptions exposées précédemment. Les orientations proposées ci-dessous ont en commun de considérer le risque comme une situation vécue, plaçant l'individu au cœur du processus d'identification d'une certaine réalité des risques sportifs. Griffet (1994) considère en effet tout activité sportive comme un «comportement compréhensible » qui détient une part de sens subjectif. L'opposition avec les définitions structurelles est claire, puisqu'il n'est plus fondamentalement question de danger. On rejoint ici le point de vue de Duclos (1987) : «le risque, c'est un style d'être au monde, un filtre herméneutique des actes, et certainement pas un attribut des choses qui attendraient, passives, leur agent». Dans cette perspective, définir les «sports à risque » implique la prise en considération de la subjectivité. 


\section{Les comportements individuels en situation dangereuse}

Comment le comportement se construit-il en situation d'incertitude, lorsque l'éventualité concrète de l'accident est bien réelle? On ne parle plus de «perception des risques", mais de perception du danger et des stratégies d'évitement mises en œuvre.

Dans une optique cognitiviste, Delignières (1991) différencie risques objectifs et subjectifs liés aux situations motrices ${ }^{8}$. Les premiers dépendent des caractéristiques intrinsèques de la situation, alors que les «risques subjectifs » renvoient au domaine psychoaffectif du pratiquant. Constatant d'une part que le risque perçu est une dimension propre à chaque individu, et d'autre part que les joueurs exercent une action et un contrôle sur l'éventualité d'un événement, ce psychologue affirme qu'il est illusoire de chercher à définir dans l'absolu le risque sportif, par essence relatif.

Selon Griffet (1994), lorsqu'il arrive au seuil de la connaissance du réel et s'avance dans un espace qui recèle sans doute du danger, le preneur de risques s'engage dans une situation sur laquelle la raison n'a plus de prise directe. Il utilise alors des procédures $^{9}$ destinées à déterminer à l'avance la nature et la succession des événements susceptibles de se produire. La lecture de cet avenir immédiat est basée sur un processus inductif de projection principalement qualitatif et généalogique, c'est-à-dire basé sur l'expérience et l'assimilation progressive du champ des issues possibles lié à chaque type de situation. Le risque est alors celui d'une erreur dans l'opération d'abstraction et le jugement produit. On en arrive à la conclusion que l'estimation du risque est un problème intime lié à la confiance, aux compétences et à la perception individuelle de la situation.

\section{Le risque : une expérience sensible}

De Léséleuc et Raufast (2005) affirment que seuls les acteurs peuvent permettre de déceler les principes définitionnels de leurs activités. Parmi ces derniers, le risque, tel qu'il est vécu et ressenti, occupe une place de première importance. L'apport de ces deux chercheurs prend racine dans l'étude des activités sportives pratiquées en montagne. Le rapport entretenu avec le risque par les grimpeurs et les alpinistes apparaît en effet comme une variable discriminante permettant de différencier ces activités ascensionnelles. Les pratiques articulant le corps et la verticalité sont ainsi considérées comme participant de l'alpinisme lorsqu'elles se fondent sur des logiques d'affrontement et de prise de risque (vertige par attirance du vide), et comme participant de l'escalade libre lorsqu'elles s'étayent sur des jeux de chute dans le vide (vertige par lâchage). Pour résumer, en alpinisme, il y a prise de risque

\footnotetext{
${ }^{8} \mathrm{Au}$ regard du préambule de cet article, il semble préférable de substituer le couple danger et risque à celui, fréquemment mobilisé, de risques objectifs et subjectifs.

9 L'individu recourt à des heuristiques d'une rationalité limitée (Duclos, 1987), lesquels lui permettent de se forger des croyances constitutives de guides pour l'action sans y consacrer trop de temps ni d'énergie.
} 
et tabou de la chute, alors qu'en escalade il y a chute et tabou du risque. C'est bel et bien le vécu de l'activité qui est premier, dans une optique phénoménologique, et non sa logique interne, jugée désincarnée.

Selon Stranger (1999), si le surf peut être qualifié d'activité sportive à risque, ce n'est pas parce qu'il occasionne des décès et des blessures graves (ce que confirment Renneker et al., 1993), mais parce qu'il est fondamentalement pratiqué en vertu du frisson éprouvé. Lequel implique occasionnellement, comme conséquence, des niveaux de risque non négligeables. Mais les surfeurs ne recherchent pas consciemment une surenchère du niveau de risque encouru ; celleci n'est que le produit de la recherche de sensations de plus en plus fortes. La prise de risque qui résulte de cet objectif est selon Stranger ce qui spécifie le surf comme activité à risque.

\section{Commentaire critique et implications méthodologiques}

Ces écrits ne sont guère encourageants dans l'optique de l'élaboration d'une définition générique des «sports à risque ». Ils ne visent d'ailleurs pas un tel objectif, réfutant la possibilité de mesurer objectivement le danger lié à une pratique sportive, et affirmant implicitement que toute pratique sportive peut être considérée comme risquée aux yeux d'un individu particulier. D'après ces chercheurs, c'est donc d'après un vécu singulier, et non au regard des traits pertinents d'une activité sportive, qu'il semble préférable de parler de situation corporellement risquée.

Ces travaux d'orientation phénoménologique et/ou post-moderne privilégient les perceptions et le ressenti individuels. Sur un plan méthodologique, le pratiquant est dès lors placé au cœur du processus d'identification des risques, à travers la réalisation d'entretiens peu directifs et d'observations directes, éventuellement participantes, comme chez Stranger ou De Léséleuc.

\section{Les « sports à risque » : une construction culturelle et sociale}

A travers ce cadre de lecture, la façon dont le corps social labellise certaines pratiques «à risque », au détriment d'autres activités non moins dangereuses, est privilégiée. Au-delà du facteur culturel, la caractérisation des «sports à risque » est également le fruit d'influences médiatiques et commerciales. Enfin, ce jeu social contribue à valoriser et légitimer certains « sports à risque », tout en condamnant de manière plus ou moins explicite d'autres expositions au danger.

\section{Désignation et légitimation sociales des « sports à risque »}

Toutes les situations porteuses de dangers n'accèdent pas au statut de risque (Gilbert, 2003). Inversement, des activités relativement sûres deviennent des risques. Une sélection sociale s'opère ; l'accession au statut de «sport à risque » semble ainsi influencée par les caractéristiques des dangers (cf. la première partie : 
gravité des écueils corporels, prises de risque plutôt que périls, etc.), mais surtout par les mises en scène médiatiques et les messages publicitaires gravitant autour de certains sports. Le caractère « émergent » d'une activité semble lui aussi constituer un facteur important de « mise en risque ».

Sans remettre en cause l'existence concrète des dangers, Kasperson et Kasperson (1996) considèrent que les interactions sociales entre leaders d'opinion, public, porte-parole d'institutions et médias sont génératrices de la visibilité sociale des risques. Le jeu médiatique, à travers la recherche de nouveauté, d'informations «percutantes », voire de sensationnalisme, joue un rôle clé dans ce processus d'amplification/atténuation de certains dangers sportifs. En s'emparant de manière privilégiée de certaines menaces «porteuses», les médias participent de l'estimation de la dangerosité des sports et du façonnage social des risques sportifs. Sans sous-estimer les dangers inhérents à cette activité, la vision de l'alpinisme comme activité à risque semble ainsi devoir autant aux récits biographiques et médiatiques d'épopées aventureuses qu'à la réalité accidentelle et aux intentions profondes de ses adeptes (Seigneur, 2004).

Ces effets médiatiques sont parfois couplés à des stratégies commerciales accentuant le brouillage des repères et des catégorisations sportives. Ainsi, le succès des notions de risque, d'aventure et d'extrême repose depuis quelques années sur un malentendu bien entretenu (Seigneur, 2004). Un marché spécifique et une définition particulière de ces notions aboutissent à leur définition sociale. Le « sensationnel assuré », sans réelle incertitude, garantissant à la fois les frissons et la sécurité, s'est imposé dans des activités telles que le saut à l'élastique, la fréquentation des parcs aventure, voire la via ferrata et l'escalade. De telles mises en scène d'épreuves contribuent à la confusion entre vertige, imaginaire du risque et danger réel. Les raids-aventure fonctionnent sur une ambiguitté comparable, en se focalisant sur l'endurance et une pseudo auto-suffisance (Barthélémy, 2002). De même, l'ambivalence de nombreuses prestations de loisirs sportifs de plein-air est soulignée par Fourré (2003) : on joue sur le frisson procuré aux clients, tout en misant sur la démonstration d'une prise en charge volontariste et performante des dangers générés par ces pratiques.

En conséquence, plusieurs sports généralement qualifiés de risqués n'apparaissent pas comme des activités impliquant beaucoup d'accidents. Le sentiment de risque associé à plusieurs activités de vertige (escalade, saut à l'élastique, voire parapente ou deltaplane), essentiellement en vertu de leurs impacts potentiels, n'a pas de traduction directe dans le bilan chiffré des victimes du sport (Majastre, 1990). En France, il y a cinq fois plus de vacanciers à la mer qu'à la montagne, mais on se noie treize fois plus (environ 600 décès par été) qu'on ne meurt en alpinisme et randonnée (Majastre, 1990). La baignade estivale n'est pourtant pas estampillée comme une pratique à risque. Dans le même ordre d'idée, la fréquentation des piscines publiques engendre entre 50 et 80 décès par an en France (Lebihain, 2002). C'est plus que les sports d'hiver (Fleury, 1999) ou l'alpinisme, et un peu moins que 
la randonnée pédestre ${ }^{10}$ (une centaine de décès « sur le coup » en moyenne chaque année) (Lefèvre et Monnier, 2002). Pour autant, la fréquentation des piscines publiques n'a rien, dans notre imaginaire collectif, de « risqué ».

L'appartenance à la catégorie des «sports à risque » n'est donc pas définie en référence au degré de dangerosité des activités, tel qu'il est avéré par l'accidentologie. Le sens de ces pratiques s'élabore à partir d'un jeu de significations sociales disponibles (Majastre, 1990). Il en va de même concernant la légitimité sociale des pratiques sportives recelant des possibilités d'écueil corporel : le risque sportif est avant tout légitimé dans les sports de nature et les disciplines non systématiquement violentes (pénétrant de préférence dans les groupes dominants et moyens), alors que les sports de combat les plus violents, essentiellement implantés en milieu populaire, bénéficient d'une acceptabilité sociale moindre (Defrance, 1983).

Dans cette perspective sociétale, définir les «sports à risque » ne saurait procéder d'une démarche intellectuelle propre à l'analyste, aussi rigoureuse soit-elle. Il s'agit en effet avant tout d'une fabrique sociale et culturelle sur laquelle l'individu a par définition peu prise, mais qu'il peut par contre tenter de comprendre. Seigneur (2004) déplore à ce propos le manque de recul de nombre de chercheurs en sciences sociales, qui deviennent dupes de la construction sociale des «sports à risque », alors que leur fonction devrait les inciter à décoder ces divers mécanismes d'imposition intellectuelle.

\section{Les représentations sociales du risque sportif}

En permettant de faire le lien entre les niveaux social et individuel, la notion de représentation sociale contribue à l'affinement de la compréhension des processus conduisant à la qualification risquée de certaines activités ou modalités de pratique sportive. L'approche en terme de représentation place au centre l'influence de l'environnement culturel et le travail cognitif qui participent d'une reconstruction du risque, dépassant sa simple perception (Joffe, 2003; Peretti-Watel, 2001). Renvoyant implicitement à la différence entre danger et risque, elle postule qu'il ne peut y avoir de risque sans sujet, individuel ou collectif, pour produire les représentations correspondantes (Coanus et al., 1999).

Les dangers liés à la pratique des sports d'hiver ont été abordés sous cet angle (Reynier et Soulé, 2002 ; Vermeir, 2003). Pour les surfeurs comme les skieurs, les éléments centraux des représentations de ces dangers sont l'avalanche, le hors-piste et la collision. Cela illustre à nouveau le fait que l'on accorde davantage d'importance à l'ampleur d'une menace qu'à sa probabilité d'apparition. L'indice de gravité des avalanches et collisions est en effet conséquent, mais la probabilité d'être enseveli sous une avalanche est minime, tout comme sont rares les cas de

\footnotetext{
${ }^{10}$ Ces valeurs absolues gagneraient certes à être ramenées au volume global de pratique, afin de témoigner de la réelle dangerosité des pratiques. Toutefois, la randonnée pédestre et la fréquentation des domaines skiables n'ont rien de confidentiel. Plus de 7,5 millions de personnes fréquentent ainsi chaque année les domaines skiables français (Soulé, 2004).
} 
collision (les chutes occasionnant 90 à $95 \%$ des secours sur les domaines skiables) (Soulé, 2004). Les thèmes de la collision, et a fortiori de l'avalanche, semblent faire l'objet d'un effet de sur-représentativité (Slovic et al., 1984) : l'importante visibilité médiatique de ces événements conduit les individus à surestimer ce type de menace (Vermeir, 2003). Enfin, de nombreux skieurs considèrent les surfeurs comme des pratiquants plus dangereux que les autres usagers des pistes (Reynier et Soulé, 2002). Cette stigmatisation semble être le fruit conjoint de descriptions médiatiques alarmistes, suite à certains accidents emblématiques, et de comportements davantage transgressifs que dangereux (Reynier et Chifflet, 1998). Dans les faits, la croyance à la forte dangerosité des surfeurs n'a jamais été confirmée par les statistiques d'accidentologie (Servoin, 1999).

Ces quelques éléments accréditent a priori la thèse de l'imposition médiatique d'une façon particulière, par certains aspects déformée, de voir les choses. Cependant, les représentations des dangers en station ne sont pas standardisées. Le type de pratique joue notamment un rôle souligné par Vermeir (2003). Ainsi, les adeptes des patinettes (snowblades) substituent la vitesse et la chute à l'avalanche et au hors-piste parmi les facteurs centraux de leur représentation du danger. Cette appréhension spécifique des risques prouve qu'il existe une reconstruction active du risque (en fonction de spécificités techniques et du vécu de l'activité, effectivement propice au type d'événements redoutés), et pas seulement une adhésion passive et uniforme à un ensemble de croyances véhiculées par les médias.

\section{Commentaire critique et implications méthodologiques}

En terme d'appréhension des risques, crier à l'irrationalité des acteurs constitue une solution de facilité. Tout l'enjeu pour le chercheur en sciences sociales consiste à comprendre la manière dont de multiples messages (médiatiques, publicitaires, mais aussi judiciaires et politiques) sont interprétés par des acteurs sociaux qui leur impriment leurs propres convictions, valeurs, connaissances et expériences. C'est de cette alchimie complexe que naît la catégorisation de certaines activités sportives, ou de certaines modalités de pratique, comme étant « à risque ».

L'enjeu notionnel dépasse ici la manière dont les chercheurs définissent le risque, pour investir la façon dont le corps social l'appréhende, à travers une réflexivité se transformant au gré des dynamiques sociales et institutionnelles. Il est cependant délicat de démontrer empiriquement la façon dont les risques sont socialement construits et sélectionnés, à travers des phénomènes de légitimation et d'amplification/atténuation. Dans cette optique, le chercheur a peu de prise sur la définition des « sports à risque », qui se joue à une toute autre échelle et le dépasse forcément. La lecture de la construction sociale et historique de la définition dominante et légitime des pratiques sportives à risque reste en partie à faire.

Le concept de représentation sociale, dont l'identification nécessite la combinaison d'entretiens non-directifs et de questionnaires, permet de saisir comment est reconstruit (et non simplement perçu) le risque à l'échelle individuelle. Sur la base de multiples messages et traitements, les acteurs réalisent la synthèse des tendances 
macro-sociales et d'une approche purement cognitive. On comprend ainsi mieux comment certaines «distorsions», qui n'en sont du reste pas vraiment, apparaissent.

\section{L'approche systémique des risques sportifs}

Les chercheurs mettant à contribution les modèles systémiques d'analyse des risques s'inscrivent dans une perspective pragmatique : comment les dangers sontils produits dans diverses situations sportives ? Quels sont les scénarios et facteurs de risque pouvant mener aux accidents sportifs ?

Dans cette perspective, l'intentionnalité des «preneurs de risque » est envisagée avec prudence. Implicitement, en effet, une démarche volontaire d'exposition au risque est généralement associée aux «sports à risque »(cf. la première partie). Ainsi, pour Penin (2004b, 196), «les sports à risque sont le lieu d'une mise en danger délibérée des pratiquants». A l'évidence, pourtant, les risques sportifs procèdent également d'une exposition au danger non sciemment recherchée, y compris de la part de sujets pusillanimes. Bref, les risques sportifs ne relèvent pas tous de la prise de risque. Le danger gagne alors à être considéré comme un phénomène latent, une production multifactorielle seulement partiellement liée aux décisions individuelles des sportifs.

\section{De l'approche monocausale à une vision systémique du risque}

On considère souvent implicitement qu'analyser les comportements individuels suffit à expliquer la production de danger. Il faut cependant prendre acte du fait que le danger résulte généralement d'une combinaison de facteurs, parfois sans lien en apparence (Dodier, 1994). Se décentrer de l'individu et de son implication exclusive dans la production de danger devient dès lors une nécessité. «A société complexe, causalité complexe (...) l'idée de cause, trop simple, est abandonnée pour celle de facteur de risque. Cela induit d'abord la pluralité des causes (il n'y a jamais un seul facteur de risque) et leur affaiblissement (un facteur de risque n'est ni nécessaire, ni suffisant). Les facteurs de risque sont par définition multiples, variés, disséminés» (Peretti-Watel, 2000). Cette modélisation du risque rejoint celles des sciences du danger (Périlhon et al., 1993), au sein desquelles le pluralisme explicatif s'est imposé de longue date.

Kaplan et Garrick (1981) distinguent à propos du risque ce qui peut arriver (scénario), les chances pour que cela se produise (probabilité) et l'ampleur des dommages éventuels (conséquences). Les conceptualisations des « sports à risque » présentées jusqu'alors sont essentiellement axées sur l'importance et la fréquence des impacts potentiels. Ce faisant, force est de constater que la première dimension du triplet évoqué par Kaplan et Garrick (1981) est peu développée. On se limite généralement à l'évocation de la nature des écueils corporels envisageables et à la présentation de quelques facteurs de danger, au détriment de l'identification plus 
poussée des scénarios pouvant les engendrer. Une question reste dès lors en suspens : quels scénarios sont susceptibles de conduire aux événements entraînant des écueils corporels ? C'est dans cette direction que des développements sont désormais proposés. Dans une optique systémique, le danger est envisagé comme une conjonction de facteurs se succédant dans le temps. L'analyse des risques sportifs vise à cerner ce faisceau de facteurs et leur enchevêtrement complexe aux différentes étapes du processus de danger.

\section{L'exemple du risque de noyade en piscine publique}

50 à 80 cas de noyade mortelle se produisent chaque année en France. Lebihain (2002) a cherché à identifier les mécanismes de survenue de ces accidents. Il apparaît que c'est l'enchaînement d'événements parfois anodins qui peut conduire au décès. Le concept d'accident est dès lors étudié sous l'aspect des faits successifs qui se conjuguent pour parvenir au drame. La chute dans l'eau d'un enfant ne sachant pas nager ne suffit par exemple pas à expliquer un décès par noyade. La non-intervention ou l'intervention tardive d'une tierce personne constitue une condition supplémentaire indispensable, elle-même liée à un défaut de surveillance qui peut avoir pour origine une surveillance inadaptée à la fréquentation, l'absence de surveillants ou un défaut d'attention. Le scénario le plus typique est le suivant : avant l'accident, l'enfant échappe à la vigilance des parents, animateurs et maîtresnageurs ; pendant l'accident, on ne voit pas l'enfant tomber à l'eau, comme on ne voit pas non plus une noyade se produire en surface, encore moins au fond $\mathrm{du}$ bassin ; après l'accident, la victime est très difficile à percevoir et reste immergée plusieurs minutes... Une seule cause ne suffit pas : que l'un des faits évoqués supra soit absent du scénario et la noyade ne survient plus.

\section{Le cas des dangers inhérents à la pratique des sports d'hiver}

Au terme d'une analyse des risques réalisée en station de sports d'hiver, Soulé (2002) a identifié plus d'une centaine de scénarios de danger (avérés ou hypothétiques) convergeant vers une quinzaine d'événements non souhaités (de la chute à la collision entre pratiquants, en passant par l'ensevelissement sous une avalanche...). Cette description de la manifestation concrète du danger, aussi indispensable soit elle, n'est pas suffisante. Il s'avère nécessaire d'approfondir l'analyse afin de cerner les phénomènes producteurs de danger situés en amont de sa traduction concrète sur le terrain.

A un deuxième niveau, le regard doit donc se déplacer du terrain vers les facteurs de danger provenant des systèmes que constituent les stations et de leurs dispositifs de sécurité. Le but devient alors d'identifier d'éventuelles lacunes apparaissant dans la façon de lutter contre le danger. Pas moins d'une trentaine de déficits sécuritaires sont ainsi apparus en station (Soulé, 2004). Les plus flagrants sont les suivants : schémas de communication préventive peu adéquats, domination du critère productiviste sur la sécurité des clients, valorisation marketing du hors-piste, faible temps accordé par les loueurs à chaque client, ouverture des pistes en conditions nivologiques et/ou météorologiques non idéales, oubli de certaines règles de 
sécurité de la part des opérateurs de terrain, etc. Par ailleurs, certaines influences provenant de l'environnement des stations sont susceptibles d'influer sur les processus de danger: influences des messages médiatiques, des évolutions technologiques, culturelles, etc. (Soulé, 2002).

Au-delà des cas particuliers des piscines publiques et des sports d'hiver, chaque pratique sportive génère des dangers particuliers qui peuvent être identifiés selon cette perspective systémique. La nature des événements, leurs impacts et les scénarios pouvant conduire à chacun d'entre eux constituent la structure du danger inhérent à la pratique d'une activité sportive. L'utilité même d'une définition des «sports à risque » se voit ainsi remise en question, puisque toute pratique peut faire l'objet d'une analyse des risques qui mettrait à jour des processus de danger spécifiques. Il ne s'agit pas d'en conclure que toutes les activités sportives sont identiquement dangereuses, chacune d'elles générant une accidentologie spécifique, à rapporter au nombre de pratiquants exposés et à leur volume de pratique.

\section{Commentaire critique et implications méthodologiques}

Les applications de l'analyse systémique des risques sportifs permettent de mieux saisir la manière dont Peretti-Watel (2001) caractérise la notion de risque : un danger sans cause, un dommage sans faute, bien que prévisible en référence aux outils statistiques et probabilistes. L'usage du singulier n'est pas anodin : chaque accident constitue en fait le symptôme d'une foule de circonstances particulières. Une nouvelle conception du lien causal doit dès lors être adoptée : à chaque danger sont en effet associés des «facteurs de risque » spécifiques dont la présence accroît la probabilité d'occurrence du risque, sans en constituer une cause nécessaire et suffisante.

Des critiques peuvent bien sûr être adressées aux visions systémiques du risque. En effet, la simultanéité des effets producteurs de danger, qui est une caractéristique de l'application de la théorie des systèmes à l'analyse du danger, renforce la contingence de l'enchaînement entre les événements et les dommages (Luhmann, 1991). Le nombre de facteurs de risque théoriquement envisageables dans un scénario de danger est incalculable et laisse le «préventologue » dubitatif : s'il identifie une cause unique ayant un effet néfaste, il lui suffit d'agir sur cette cause pour supprimer l'effet. Mais si l'effet a une multitude de causes partielles (et potentielles), il n'est plus possible de les prévenir de façon exhaustive. Sur quelles causes intervenir en priorité lorsque la notion de risque les disperse?

Sur un plan méthodologique, cette perspective pragmatique implique un recueil de données élargi, permettant de recenser l'ensemble des facteurs contribuant aux scénarios accidentels. Le maximum d'acteurs aux profils, statuts et fonctions différents doit être consulté, afin de bénéficier de leur expérience (relative aux événements passés), de leur «créativité négative» (en termes de scénarios envisageables), et d'atteindre in fine une certaine représentativité fonctionnelle (plutôt que statistique) du système porteur de risques. Il convient également de multiplier les sources documentaires : bilans accidentologiques, mains-courantes, 
articles de presse, expertises techniques, décisions judiciaires, etc. Le postulat étant celui du pluralisme explicatif, l'enjeu est de se défaire des conceptions simplistes du risque (souvent monocausales), tout en relativisant les effets d'autorité induits par certaines expertises unilatérales, fréquemment dépendantes d'intérêts particuliers.

\section{CONCLUSION}

Des tentatives d'objectivation structurelle aux perspectives relativistes de la psychologie cognitive et de la phénoménologie, en passant par les approches culturaliste ou systémique, l'existant est varié et d'une indéniable richesse. Du coup, la proposition d'une synthèse du paysage terminologique dressé constitue une entreprise difficile, et au regard du degré d'éclatement des usages et définitions recensés, la notion de «sport à risque » peut sembler fragile ${ }^{11}$.

Une autre interprétation de cette diversité consiste à considérer le risque et les « sports à risque » comme des notions polythétiques (Needham, 1975). On ne peut dès lors espérer les définir que de manière relative et arbitraire. A travers un processus d'épuration, la connaissance scientifique considère pourtant comme impérative la transformation des notions de type polythétique en concepts de type non polythétique (Boudon, 1990). D'où le malaise ressenti dans la communauté scientifique devant les usages pluriels, parfois peu argumentés, des notions débattues dans le cadre de cet article. Les contradictions soulevées passent généralement pour un problème, justifiant des tentatives plus ou moins heureuses de formalisation unificatrice, s'accompagnant parfois de réquisitoires à l'encontre de points de vue non partagés. Si certaines de ces tentatives terminologiques font preuve de pondération et de relativisme, un puissant biais est sous-jacent à d'autres quand la catégorie des « sports à risque » est interprétée à travers l'a priori qui veut que l'on puisse aboutir à une définition claire et partagée. D'où l'entreprise illusoire visant à aboutir à la bonne définition du risque sportif et l'étonnement qu'une notion puisse jouer un rôle important dans le discours scientifique sans se voir associer une définition précise (Boudon, 1990). Les appels de Collard (1997 ; 1998) à davantage de rigueur conceptuelle, suivis de la proposition d'une définition visant à objectiver ce qu'il faut entendre par « sport à risque », illustrent cette position.

L'entreprise visant à aboutir à «la » bonne définition du risque sportif ou des « sports à risque » semble vaine. Comme le précisent Duchastel et Laberge (1999), prétendre parvenir à une définition générique (censée constituer le reflet de la réalité) contraint à opérer des rapprochements entre des situations relevant de dynamiques extrêmement différentes. Quel est le degré de similitude entre les modalités d'exposition au danger du rugbyman professionnel, du pratiquant

\footnotetext{
${ }^{11}$ Elle l'est aussi aux yeux des pratiquants d'activités archétypales du risque sportif (parachutisme, B.A.S.E. jump, freeride, alpinisme et parapente), qui rejettent unanimement cette appellation, l'assimilant à un dessaisissement irresponsable de leur sort, alors qu'ils sont au contraire désireux d'afficher la cohérence de leur ligne de conduite et la rationalité de leurs choix (Penin, 2004a).
} 
ponctuel du saut à l'élastique et du nageur en piscine publique ? Aucune standardisation lexicale n'est à l'évidence souhaitable. La description réaliste d'un champ conceptuel consiste surtout à reconnaître sa perpétuelle mobilité (Passeron, 2006). Se distancier d'une position naturaliste ne revient ni à renoncer à l'usage de la catégorie de «sport à risque ", ni à rendre acceptable son utilisation comme « concept-carrefour»; il s'agit d'une invitation à concevoir ce terme comme structurant activement des réalités sociales singulières. Dans cette perspective, la diversité rencontrée ne constitue pas un problème, devenant la simple preuve d'une inscription disciplinaire, paradigmatique et théorique.

La présentation des liens existant entre approche notionnelle et entrée méthodologique montre qu'un principe constructiviste orchestre la production de connaissances sociologiques. La fabrique méthodologique est indexée au paradigme de référence du chercheur, qui, en posant une définition de l'objet lors de la phase de construction de son modèle d'analyse, s'oriente déjà vers les outils adéquats de collecte des données (Quivy et Van Campenhoudt, 1995). On souligne ainsi l'absence de neutralité, et ce dès l'étape de la définition des concepts, pourtant considérée dans bien des cas comme un passage obligé sans grandes conséquences ultérieures. Dans les faits, ce travail propédeutique, pour peu qu'il soit effectué sérieusement, influence et conditionne pourtant l'intégralité du processus de recherche.

\section{REFERENCES}

Arènes, J., Janvrin, M.P. et Baudier, F. (Eds.) (1998). Baromètre santé jeunes 97/98. Vanves : C.F.E.S.

Babbie, E. (1998). The practice of social research. Belmont : Wadsworth Publishing.

Baddeley, M. (Ed) (2003). Sports extrêmes, sportifs de l'extrême : la quête des limites. Genève : Georg Editeur.

Bajoit, G. (2003). Le Changement social. Approche sociologique des sociétés occidentales contemporaines. Paris : Armand Colin.

Barthélémy, M. (2002). L'engouement pour les raids-aventure ou la société du risque transfigurée par le destin. Sociétés. Revue des Sciences Humaines et Sociales, 77, 80-97.

Baudry, P. (1991). Le corps extrême. Approche sociologique des conduites à risque. Paris : L'Harmattan .

Beck, U. (1999). Le cœur de la modernité. In Le Monde des Débats, novembre, 12- 
15.

Boudon, R. (1990). L'art de se persuader des idées douteuses, fragiles ou fausses. Paris : Fayard.

Bouet, M. (1968). La signification du sport. Paris : Editions Universitaires.

Bourdeau, P. (1996). Le statut ambigu du risque dans les sports de montagne. Communication au Colloque du Salon d'Aménagement de la Montagne, Grenoble.

Bozonnet, J.P. (1992). Des monts et des mythes. L'imaginaire social de la montagne. Presses Universitaires de Grenoble : Grenoble.

Brohm, J.M. (1987). Urgences sportives, l'extrême limite. Actions et recherches sociales, 2.

Brohm, J.M. (Ed) (1986). Quel corps ? Paris : Editions de la passion.

Coanus, T., Duchêne, F. et Martinais, E. (1999). Les relations des gestionnaires du risque urbain avec les populations urbaines. Critique d'une certaine idée de la « communication ». Annales des Mines, janvier, 8-13.

Collard, L. (1997). Approche sociologique des sports à risque. STAPS, 44, 83-96.

Collard, L. (1998). Sports, enjeux et accidents. Paris : PUF.

De Léséleuc, E. et Raufast, X. (2005). Du risque en sports de nature : une approche socio-psychanalytique. Revue Européenne de Management du Sport, 12, 237-258.

Defrance, J. (1983). La tentation de l'accident. Le risque en sport et sa légitimation. Culture technique, 11, 317-321.

Delignières, D. (1991). Risque perçu et apprentissage moteur. In Apprentissage moteur : rôle des représentations. Paris : Editions EPS, 157-171.

Dodier, N. (1994). Causes et mises en cause. Innovation sociotechnique et jugement moral face aux accidents du travail. Revue Française de Sociologie, 35, 251-281.

Douglas, M. (1985). Risk acceptability according to the social sciences. New York : Russel Sage Foundation.

Douglas, M. (1987). How institutions think. London : Routledge.

Duchastel, J. et Laberge, D. (1999). La recherche comme espace de médiation interdisciplinaire. Sociologie \& Sociétés, 31, 1, 63-76.

Duclos, D. (1987). La construction sociale du risque : le cas des ouvriers de la 
chimie face aux dangers industriels. Revue Française de Sociologie, 28, 17-42.

Fleury, B. (1999). Système National d'Observation de la Sécurité en Montagne. Cahiers du C.S.S.M., 11, 14-30.

Fourre, C. (2003). Le secteur des stages outdoor : la professionnalisation des consultants d'entreprise. Revue Européenne de Management du Sport, 9.

Giddens, A. (1987). La constitution de la société. Paris : PUF.

Gilbert, C. (2003). La fabrique de Risques. Cahiers Internationaux de Sociologie, CXIV, 55-72.

Griffet, J. (1994). Décision, risque, émotion. Science \& Motricité, 23, 3-12.

Hahn, A., Eirmbter, W.H. et Jacob, R. (1994). Le SIDA : savoir ordinaire et insécurité. Actes de la Recherche en Sciences Sociales, 104, 81-89.

Howe, D.P. (2001). An ethnography of pain and injury in professional rugby union. The Case of Pontypridd RFC. International Review for the Sociology of Sport, 36, 289-303.

Jackson, D.N., Hourany, L. et Vidmar, N.J. (1972). A four-dimensional interpretation of risk taking. Journal of Personality, 40, 483-501.

Jardine, G.J. et Hrudey, S.E. (1997). Mixed messages in risk communication. Risk analysis, 17, 4, 489-498.

Joffe, H. (2003). Risk : from perception to social representation. British Journal of Social Psychology, 42, 55-73.

Kaplan, S. et Garrick, J. (1981). On the quantitative definition of risk. Risk Analysis, 1, 1.

Kaplan, S. (1997). The words of risk analysis. Risk Analysis, 17, 4, 407-417.

Kasperson, R.E. et Kasperson, J.X. (1996). The social amplification and attenuation of risk. The Annals of the American Academy of Political and Social Science, 545, 95-105.

Kates, R.W. et Kasperson, R.E. (1983). Comparative risk analysis of technological hazards (a review). Proceedings of the National Academy of Sciences, 80, 70277038 .

Kay, J., et Laberge, S. (2002). Mapping the field of «AR » : Adventure Racing and Bourdieu's concept of field. Sociology of Sport Journal, 19, 25-46. 
Kervern, G.Y., et Rubise, P. (1991). L'archipel du danger. Introduction aux cindyniques. Paris : Economica.

Kuhn, T. (1972). La Structure des révolutions scientifiques. Paris : Flammarion.

Kunreuther, H. et Slovic, P. (1996). Challenges in risk assessment and risk management. The Annals of the American Academy of Political and Social Science, 545. London : Sage Publications, 1-183.

Kusz, K. (2003). BMX, extreme sports, and the white male backlash. In R.E. Rinehart, et S. Sydnor, (Eds.), To the extreme. Alternative sports, inside and out. (pp. 153-175). Albany: State University of New York press.

Lavigne, J.C., Blancher, P., Peguet, P., Maccio, N., Navarro, A. et Caillot, R. (1989). L’univers sémantique du risque. Préventique, 25, 19-32.

Le Breton, D. (2002). Conduites à risque. Paris : PUF.

Lebihain, P. (2002). Une approche de la sécurité dans les espaces de loisirs. Le cas des piscines publiques. Cahiers Espaces, 73, 99-117.

Lefèvre, B. et Monnier, A. (2002). L'étude de la mortalité des sports de montagne : entre accidentologie et démographie. Communication lors du 22ème colloque national de démographie, Amiens, 14-16 mai.

Luhmann, N. (1991). Soziologie des risikos. Berlin-New York : De Gruyter.

Lupton, D. (1999). Risk. New York : Routledge.

Majastre, J.O. (1990). Risque et incertitude. In Actes du colloque Vertiges, sports à risque et toxicomanies. Marseille : A.M.P.P., 27-33.

Midol, N. (1993). Cultural dissents and technical innovations in the "whiz" sports. International Review for the Sociology of Sport, 28, 23-32.

Mun, S. (2004). The perception of risk in sport activities. Doctoral Dissertation, Florida State University, College of Education.

Needham, R. (1975). Polythetic classification: convergence and consequences. Man, 10, 349-369.

Palmer, C. (2002). "Shit happens": the selling of risk in extreme sport - Interlaken and Everest tourist tragedies. The Australian Journal of Anthropology, 13, 323-336.

Parlebas, P. (1981). Contribution à un lexique commenté en sciences de l'action motrice. Paris : INSEP. 
Passeron, J.C. (2006). Le raisonnement sociologique. Un espace non poppérien de l'argumentation. Paris : Albin Michel.

Penin, N. (2004a). Prises de risques sportifs : saisir des modes d'engagement sexués. Actes du Colloque Acteur, risque et prise de risque à l'épreuve des sciences sociales, 338-344. (www.univlille1.fr/clerse/site clerse/PDF/pdfnews/colloque2004/rapport risque final.pdf)

Penin, N. (2004b). «L'amour du risque ». Modes d'engagements féminins dans les pratiques sportives à risque. STAPS, 66, 195-207.

Peretti-Watel, P. (2000). Sociologie du risque. Paris : Armand Colin.

Peretti-Watel, P. (2001). La société du risque. Paris : La Découverte.

Périlhon, P., Penalva, J.M., Coudouneau, L., Dos Santos, J., Lesbats, M. et Dutuit, Y. (1993). Développement d'un modèle de référence en sciences du danger. $1^{e r}$ colloque international des Cindyniques, Cannes.

Pociello, C. (1995). Les cultures sportives. Pratiques, représentations et mythes sportifs. Paris : PUF.

Quivy, R. et Van Campenhoudt, L. (1995). Manuel de recherche en sciences sociales. Paris : Dunod.

Renn, O. (1998). Three decades of risk research : accomplishments and new challenges. Journal of Risk Research, 1, 49-71.

Renneker, M., Star, K., et Booth, G. (1993). Sick surfers ask the surf docs and Dr Geoff. Palo Alto : Bull Publishing Company.

Reynier, V. et Chifflet, P. (1998). Pratiques sportives, représentations sociales et comportements territoriaux : les pratiquants des stations de sports d'hiver. Cahiers Internationaux de Psychologie Sociale, 38, 84-97.

Reynier, V. et Soulé, B. (2002). Les représentations sociales des risques chez les pratiquants des stations de sports d'hiver. $1^{\text {er }}$ Congrès de la Société de Sociologie du Sport de Langue Française : Sport et Vie sociale, transformations, ruptures et permanences, Toulouse, 28-30 octobre.

Rinehart, R.E., et Sydnor, S. (Eds) (2003). To the extreme. Alternative sports, inside and out. Albany : State University of New York press.

Rotillon, G. (2003). L'alpinisme et l'escalade dans l'économie des loisirs. In M. Baddeley (Ed), Sports extrêmes, sportifs de l'extrême. La quête des limites (pp.141157). Genève: Georg éditeur. 
Schrader, M.P. et Wann, D.L. (1999). High-Risk Recreation: The Relationship Between Participant Characteristics and Degree of Involvement. Journal of Sport Behavior, 22, 426-441.

Seigneur, V. (2004). L'acteur preneur de risques : des figures comportementales multiples ? Les problèmes de définition du risque. Actes du Colloque Acteur, risque et prise de risque à l'épreuve des sciences sociales, 352-359. (www.univlille1.fr/clerse/site clerse/PDF/pdfnews/colloque2004/rapport risque final.pdf)

Servoin, F. (1999). L'introuvable responsabilité des surfeurs. Tourisme \& Droit, 10, 8-9.

Slovic, P., Fischhoff, B. et Lichtenstein, S. (1984). Modeling the societal impact of fatal accidents. Management Science, 30, 464-474.

Soulé, B. (2002). Analyse socio-technique des risques en station de sports d'hiver. Annales des Ponts et Chaussées, 104, 65-74.

Soulé, B. (2004). Sports d'hiver et sécurité. De l'analyse des risques aux enjeux de leur gestion. Paris : L'Harmattan.

Soulé, B. (2006). Les «sports extrêmes»: analyse terminologique d'une caractérisation sportive à succès. Science \& Motricité (sous presse).

Stranger, M. (1999). The aesthetics of risk. A study of surfing. International Review for the Sociology of Sport, 34, 3, 265-276.

Vassort, P. (2003). Sport. In Dictionnaire des risques. Paris : Armand Colin, 364366.

Vermeir, K. (2003). Le risque sur les domaines skiables alpins. Analyse des représentations sociales des pratiquants. Mémoire de D.E.A. STAPS, Université Joseph Fourier, Grenoble. 